\section{Verhüten? Logo ...}

Kontrazeption ist in der pädiatrischen Praxis ein Thema - sollte es auch. Denn Pädiater haben ein gutes Vertrauensverhältnis zu ihren Patientinnen, in dem "auch intime Fragen möglich sind“. Verhütung sollte deshalb laut der Gynäkologin Dr. Marlene Heinz aus Berlin, Vorsitzende der Arbeitsgemeinschaft Kinder- und Jugendgynäkologie e.V., ,aktiv angesprochen werden, da junge Mädchen dies kaum von sich aus tun." Dabei ist es wichtig, unbegründete Sorgen vor Kontrazeptiva zu nehmen: „Das erste Mal Verhüten ist mit vielen Ängsten und Erwartungen, wie einfache Anwendung und Zugänglichkeit, verbunden." Außer die jugendliche Patientin aufzuklären, gilt es, sie über geeignete Verhütungsmöglichkeiten zu beraten. Mittel der Wahl für diese Altersgruppe sind dank

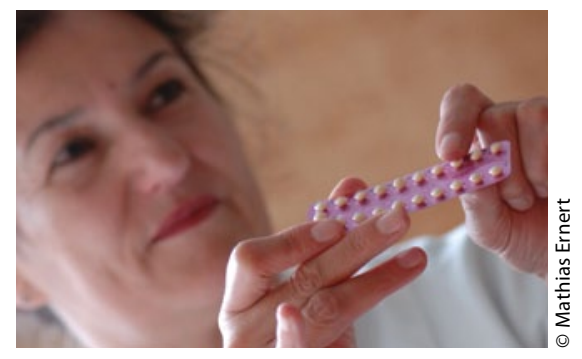

hoher Sicherheit und wenig Nebenwirkungen orale EE-Gestagen-Kombinationen, die Mikropillen. Orale Gestagenpräparate, Minipillen, sind dagegen „nur bei Östrogenkontraindikation angezeigt", so Heinz. Hormonpflaster und -ringe wären gute Alternativen, finden jedoch zu wenig Akzeptanz. Nur bedingt geeignet sind Hormonspiralen. Implantate sind nur bei sozialen Indikationen wie Drogen- oder Alkoholmissbrauch angezeigt. Gänzlich abzuraten ist von
Depotpräparaten wie der Dreimonatsspritze - unter anderem deshalb, weil sie die Knochendichte herabsetzen und zu Gewichtszunahme bzw. Akne führen. Bei der Beratung sind laut der Berliner Gynäkologin unbedingt auch die individuelle Lebenssituation und etwaige Erkrankungen zu berücksichtigen. So sollten etwa bei schweren Herzkrankheiten, Hypertonie sowie akuten Lebererkrankungen keine Östrogene und auch bei erhöhtem Thromboserisiko einzig Gestagen-Monopräparate eingesetzt werden. Die Verordnung der Kontrazeptiva obliegt Gynäkologen, so Heinz, „die auch jugendgerecht arbeiten“. Sie sind unter anderem auf der Internetseite der Arbeitsgemeinschaft www.kindergynaekologie.de zu finden.

Birgit Frohn

Heinz M. Welche Probleme in der Kontrazeptionsberatung bei Jugendlichen kann es für den Kinder- und Jugendarzt geben?

\title{
Wie viele Kinder sind psychisch gestört?
}

Psychische Störungen bei Kindern und Jugendlichen haben eine hohe Relevanz - ähnlich hoch wie Adipositas. Mithilfe der BELLA-Studie möchte das RobertKoch-Institut mehr über das seelische Wohlbefinden und Verhalten von Kindern und Jugendlichen herausfinden. Die Ergebnisse zu den psychischen Auffälligkeiten wurden von der Studienleiterin, Prof. Dr. Ulrike Ravens-Sieberer, vom Universitätsklinikum HamburgEppendorf vorgestellt: Nach den Ergebnissen der BELLA-Studie leiden dabei 9,7\% der Kinder sehr wahrscheinlich an einer psychischen Störung. Bei etwa $12,2 \%$ besteht möglicherweise eine psychische Auffälligkeit. Insgesamt liegen somit bei 21,9\% der Studienteilnehmer zumindest Hinweise auf psychische Auffälligkeiten vor. „Ich möchte dies ausdrücklich relativieren: Wir haben eine ziemlich stabile Rate von ca. $10 \%$ von Kindern und Jugendlichen, bei denen wir mit hoher Wahrscheinlichkeit von einer psychischen Störung sprechen können“, erklärte Ravens-Sieberer. Daneben gibt es einen Anteil von Kindern, bei denen es notwendig ist, genauer abzuklären und sie und ihre Familien stärker zu begleiten. Jungen sind mit 10,9\%

häufiger betroffen als Mädchen (8,4\%). Die häufigsten psychischen Erkrankungen sind Ängste, die $10 \%$ der Erkrankungen ausmachen und vor allem jüngere Kinder betreffen. Störungen des Sozialverhaltens sind mit 7,6\% vertreten. Depressionen kommen bei 5,4\% vor.

Eines der überraschendsten Ergebnisse der BELLA-Studie war für Ravens-Sieberer, dass die Zahlen stark vom sozioöko-

\section{Fertilität wirksam schützen}

Die Fertilität ist bei Malignomen im Kindes- und Jugendalter stark gefährdet. „Das Risiko des Gonadenverlustes durch Chemotherapie ist hoch, bei Radiotherapie drohen gar hundertprozentige Schäden der Gonaden“, weiß Dr. Patricia Oppelt aus ihrer Praxis im Universitätsklinikum Erlangen. Auch die Gebärmutter ist gefährdet. Die Protektion der Fertilität bei Mädchen ist mithin essenziell - die Information darüber indessen absolut unzureichend, beklagte Oppelt. Umso prekärer, als dass es inzwischen einige etablierte Methoden gibt, die den jungen Krebspatientinnen ihren Kinderwunsch nomischen Status der Familien abhängig waren. Kinder aus Familien mit einem niedrigen sozioökonomischen Status waren häufiger von psychischen Störungen betroffen. Außerdem wirkten sich Konflikte in der Familie, elterliche Belastungen und die psychische Gesundheit der Eltern stark auf die Entwicklung einer psychischen Auffälligkeit aus. $n z$

Ravens-Sieberer U. Verhaltensauffälligkeiten und psychische Probleme bei Kindern und Jugendlichen in Deutschland 